\title{
Immunofluorescence Microscopy And Environmental Scanning Electron Microscopy Analysis Of Stemonitis flavogenita
}

\author{
Robert K. Pope*, Jeannie T.B. Collins**, Scott P. Collins \\ *Indiana University South Bend, 1700 Mishawaka Avenue, South Bend, IN 46634 \\ **University of Southern Indiana, 8600 University Boulevard - Evansville, IN 47712
}

Stemonitis flavogenita, an acellular slime mold displays protist, fungal and animal attributes. These properties make classifying organisms such as Stemonitis difficult. The spores germinate, and give rise to either haploid flagellated swarm cells, or haploid myxamoebae. The myxamoebae resemble protists. When the environment changes, the swarm cells may resorb their flagella and become myxamoebae. These cells will fuse to make a new diploid plasmodium, which will move around assimilating nutrients in moist places such as leaf litter or on decaying logs [1]. These single celled, multinucleated plasmodia can be very large, reaching diameters of one to two feet, if conditions permit. The large plasmodium pumps protoplasm back and forth through the cell in specialized channels to transport nutrients and oxygen throughout the organism (Fig. 1). This appearance of vessels pumping protoplasm resembles a rudimentary vascular system, and initially lead to potential classification as an animal. However it is the formation of spores that ultimately leads to the classification as fungi.

The plasmodium is initially referred to as a microplasmodium because of its size, and then when visible to the eye without the aid of a microscope it is a plasmodium. In Stemonitis, the cell grows and becomes an assimilative aphanoplasmodium, which contains a thin network of anastomosed veins [2]. The movement of the cytoplasm in these veins may be directly dependent on the cytoskeleton. Staining of kinesin shows localization to veins in the aphanoplasmodium, and high concentrations within membrane ruffles (Fig. 2).

When conditions become dry, the plasmodium will change to the coralloid stage, condensing and moving away from the moist substrate prior to forming sporangia. The sporangia form by pumping protoplasm upward and forming a stalk (Fig. 3). Protoplasm is then pumped upward on top of the stalk and will cleave to form spores. The process of moving the protoplasm up the stalk involves numerous cytoskeletal elements. Figure 4 shows localization of the motor protein kinesin in a developing sporangium, indicating the movement of cellular components on microtubules. Spores produced allow the organism to survive unfavorable conditions, because the spores are very resistant to desiccation, even for long periods of time [1].

The use of environmental scanning electron microscopy is useful for analysis of many microorganisms [3], including the stages of the myxomycete life cycle, as is evident by the examination of cross sections of freshly formed sporangia (Figs. $5 \& 6$ ). The uncoated samples show the spacing and distribution of spores and capletial threads inside the sporangium. The outer covering (peridium) is visible at the bottom of Figure 5. This is especially useful in looking for calcium deposition that is common in many myxomycetes after the formation of sporangia.

\section{REFERENCES}

1. Alexopoulos, C. J., and Mims, C. W. Class Myxomycetes. Introductory Mycology, 3rd ed.; Wiley \& Sons: New York, 1979; pp 61-98.

2. Collins, S. P. Cytoplasmic Elements Associated with Structure and Cytoplasmic Streaming in Stemonitis flavogenita. M.S. Thesis, University of Southern Mississippi, Hattiesburg, MS, 1994.

3. Collins, SP, Pope, RK, Scheetz, RW, Ray, RI, Wagner, PA, Little, BJ (1993) Advantages of environmental scanning electron microscopy in studies of microorganisms. Micros. Research Tech. 25: 398-405. 


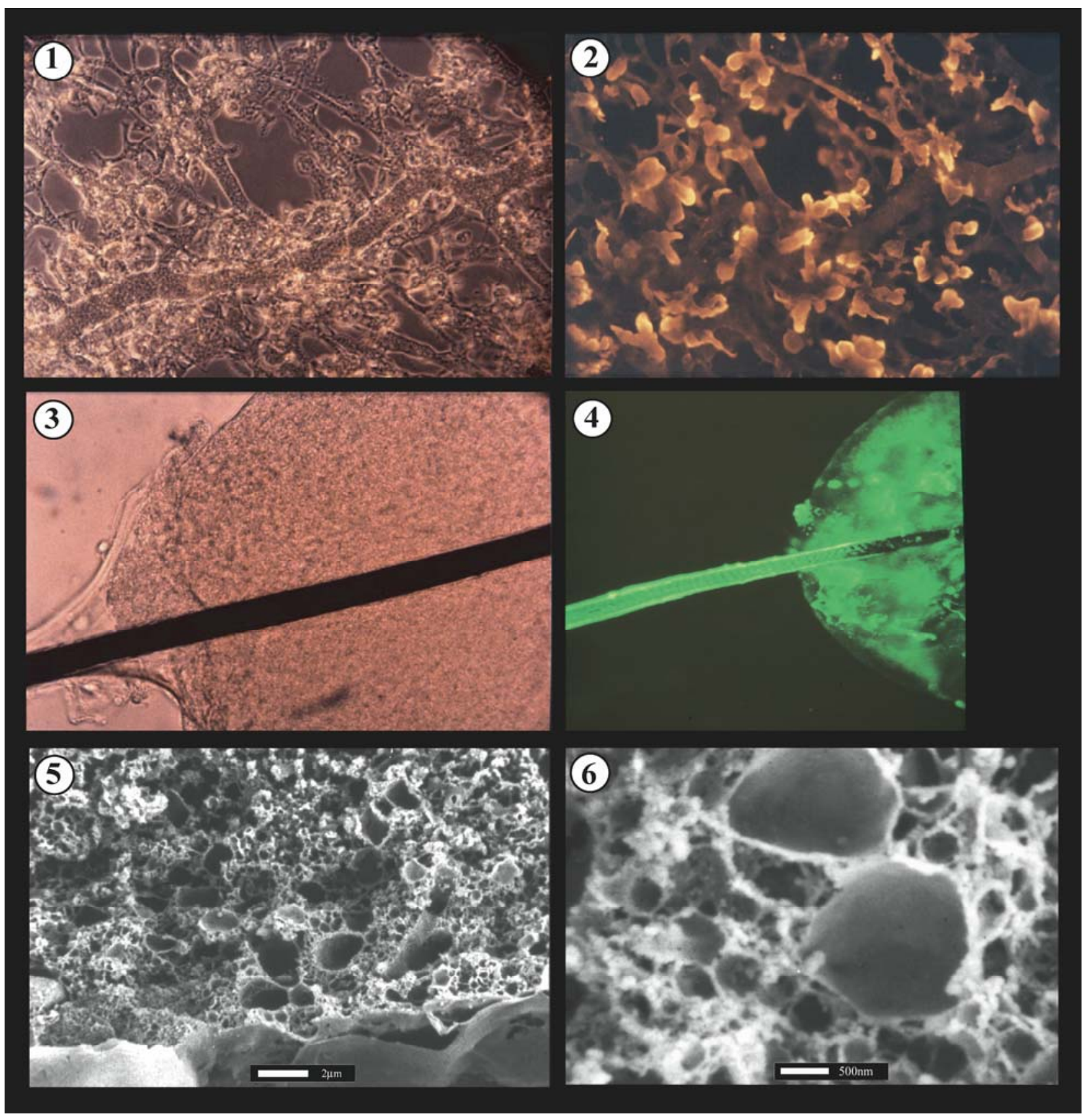

Figure 1. Phase image of fixed aphanoplasmodium on coverslip. The plasmodium was grown on oatmeal agar plates

Figure 2. TRITC anti-kinesin staining in the same plasmodium as in Figure 01. Note the abundant staining at each pseudopod that is being extended.

Figure 3. Phase image of developing sporangium that has been pressed under a coverslip

Figure 4. Bodipy ${ }^{\circledR}$ anti-kinesin staining of a developing sporangium.

Figure 5. ESEM image of mature sporangium. The peridium is visible at the bottom of the figure.

Figure 6. ESEM image of mature sporangium. 